\title{
Large Tunable 16-Tupled Millimeter Wave Generation Utilizing Optical Carrier Suppression With a Tunable Sideband Suppression Ratio
}

\author{
Asha* and Sandeep Dahiya \\ Department of ECE, Faculty of Engineering and Technology, BPS Women University, Khanpur Kalan, Sonepat, India
}

Coping up with the rising bandwidth demands for $5 \mathrm{G}$ ultra-high speed applications, utilizing millimeter (MM) wave spectrum for data transmission over the radio over a fiberbased system is the ideal approach. In this study, a highly conversant and spectrally pure photonic generation of a 16-tupled MM wave signal using a series-connected DD-MZM with a lower modulation index, a splitting ratio, and a wider tunable range is presented. A $160-\mathrm{GHz}$ MM wave is generated through a double sideband optical carrier suppression technique having an optical sideband suppression ratio (OSSR) of $69 \mathrm{~dB}$ and a radio frequency sideband suppression ratio (RSSR) of $40 \mathrm{~dB}$. However, the OSSR and the

OPEN ACCESS

Edited by:

Santosh Kumar,

Liaocheng University, China

Reviewed by:

Sharda Vashisth,

The NorthCap University, India

Dhramendra Yadav,

Bikaner Technical University, India

*Correspondence:

Asha

ashabalhara89@gmail.com

Specialty section:

This article was submitted to

Optics and Photonics,

a section of the journal

Frontiers in Physics

Received: 25 July 2021 Accepted: 17 August 2021 Published: 18 October 2021

Citation:

Asha and Dahiya S (2021) Large Tunable 16-Tupled Millimeter Wave Generation Utilizing Optical Carrier Suppression With a Tunable Sideband

Suppression Ratio.

Front. Phys. 9:747030.

doi: 10.3389/fphy.2021.747030 RSSR are tunable with values greater than $15 \mathrm{~dB}$ when the modulation index (M.I.) varies from 2.778 to $2.873, \pm 8^{\circ}$ phase drift, and a $15-\mathrm{dB}$ enhancement in the OSSR with a wider nonideal parameter variation range giving acceptable performance can be seen in the model as compared with previous research works.

Keywords: millimeter wave generation, radio over fiber system, 16 tupling, OSSR, RSSR, filterless, optical carrier suppression

\section{INTRODUCTION}

For supporting multi gigabit per second (gbps) wireless connectivity and resolving spectrum shortage, 5G uses higher frequencies from an $\mathrm{MM}$ wave range $(30-300 \mathrm{GHz})$ for signal transmission. However, the generation and transmission of MM wave signals is a troublesome process as these high-frequency signals apart from providing a wide bandwidth limits the transmission distance due to propagation losses. So, in order to cope up with this issue, the MM wave signal is first generated by modulating a low-frequency electrical signal over an optical carrier coming from a light source at a central station (CS) and then transmitting it to the base station (BS). This technique is named as millimeter wave-based radio over the fiber (i.e., MMoF) system. This fiber-based system provides low insertion and transmission losses with being immune toward electromagnetic interference.

The basic requirement for designing an efficient MMoF system is to modulate the RF signal for generating a desired $M M$ wave signal with suppressing all other unwanted signals. The MM waves can be generated in two domains: 1) electrical and 2) optical or photonic. However, MM wave signal generation in a photonic domain is best suited in comparison to their electrical counterparts due to lower phase noise, lower equipment requirement, higher spectral purity, a wider tunable range, and a larger transmission distance [1]. Several different techniques have been discussed in the past for photonic MM wave signal generation which include a direct 
TABLE 1 | Literature review of frequency 16-tupled MM wave generation based on different parameters.

\begin{tabular}{|c|c|c|c|c|c|c|c|c|c|}
\hline \multirow{3}{*}{$\begin{array}{l}\text { Author } \\
\text { (Year) }\end{array}$} & \multirow[t]{3}{*}{ Working Principle } & \multicolumn{8}{|c|}{ Parameters } \\
\hline & & \multirow{2}{*}{$\begin{array}{l}\text { OSSR } \\
\text { (dB) }\end{array}$} & \multirow{2}{*}{$\begin{array}{l}\text { RSSR } \\
\text { (dB) }\end{array}$} & \multirow[t]{2}{*}{ Filters } & \multirow{2}{*}{$\begin{array}{l}\text { Modulation } \\
\text { index }\end{array}$} & \multirow{2}{*}{$\begin{array}{c}\text { Cost } \\
\text { effective }\end{array}$} & \multirow{2}{*}{$\begin{array}{l}\text { Power } \\
\text { efficient }\end{array}$} & \multirow[t]{2}{*}{ Tunability } & \multirow{2}{*}{$\begin{array}{c}\text { O/P freq } \\
\text { Generated } \\
\text { (GHz) }\end{array}$} \\
\hline & & & & & & & & & \\
\hline Zihang Zhu [6] (2015) & Two-cascaded dual parallel MZM & 21.5 & 38 & No & 2.265 & No & No & Low & 60 \\
\hline $\begin{array}{l}\text { K. Esakki Muthu [10] } \\
(2016)\end{array}$ & $\begin{array}{l}\text { Parallel combination of two- } \\
\text { cascaded MZM }\end{array}$ & 20 & 28 & Yes & 3.14 & Yes & Yes & Low & 60 \\
\hline M. Baskaran [14] (2018) & Cascaded MZM & 54 & 42 & No & 2.827 & Yes & No & High & 80 \\
\hline Dongfei Wang [13] (2019) & Two-cascaded MZM & 31.35 & 24.11 & Yes & 7.59 (very high) & Yes & Yes & Low & 160 \\
\hline Aasif Bashir Dar [9] (2020) & Cascaded parallel MZM & 64 & 31 & No & $2.79-2.86$ & No & No & Low & 80 \\
\hline Proposed Scheme (2021) & Series MZM configuration & 69 & 40 & No & $2.778-2.873$ & Yes & Yes & High & 160 \\
\hline
\end{tabular}

modulation [1], an external modulation [2], optical heterodyning [3], Stimulated brillouin scattering (SBS) [4] etc.

Among the above-stated techniques, the generation method utilizing an external modulation through a Mach-Zehnder Modulator (MZM) is the most reliable one as it offers optical harmonics generation with a higher frequency multiplication factor (FMF), stability, and greater tunability as mentioned in our previous review study in [5]. When an RF signal drives an external modulator, several optical harmonics are generated due to its nonlinear transfer function. By the beating of these harmonics at photodetector, MM wave signals are generated. The main technical challenge in the frequency multiplication (FM) approach is generating required optical harmonics while eliminating unwanted optical harmonics with higher conversion efficiency. So far, FMF of 2, 4, 6, 8, 10, 12, 14, and 16 have been proposed. However, MM wave generation utilizing a high FMF is quite efficient as it eliminates the requirement of high-frequency operated local oscillator (LO) operating at CS.

Recently, several generation techniques have been reported with a 16 FMF utilizing a two-cascaded dual parallel MZM configuration [6-8], parallel combination of two cascaded MZM [9], cascaded parallel MZM [10, 11], and a feedforward modulation [12]. Dongfei wang [13] proposed a generation method with a two-cascaded MZM but with a higher modulation index $(>>7)$ requiring a high voltage radio frequency signal which in turn increases the system sensitivity. These schemes employ a filter for generating desired harmonics which not only increases system complexity but also reduces its tunability range and conversion efficiency. A filterless method has been proposed by the authors of reference [14] using cascaded MZM with an infinite extinction ratio which is not practically realizable. These techniques lead to partial cancellation of undesired harmonics and thus provide a lower sideband suppression ratio (SBSR) at the transmitting side leading to detect unwanted MM waves which deteriorate the system performance and also lower the spectral purity that is not desired for MM wave applications. The detailed review of these configurations is provided in Table $\mathbf{1}$.

In the present study, a cost-effective and spectrally pure 16-tupled $\mathrm{MM}$ wave frequency is generated by completely suppressing all the other undesired harmonics except eighth-order sideband (SB) using a series connection of a quad dual drive MZM (DD-MZM) and electrical phase shifters for optimizing biases of MZM and enhancing the OSSR and the RSSR by utilizing a lower modulation index and the extinction and the splitting ratio. A Theoretical analysis along with designing a simulation model is also carried out for understanding the cancellations of all other harmonics. The OSSR of $69 \mathrm{~dB}$ and the RSSR of $40 \mathrm{~dB}$ are obtained. The effectiveness of the 16-tupled generated MM wave is assessed by analyzing the effect of the MZM extinction ratio, the splitting ratio, the modulation index, and modulating frequency of the RF signal on the OSSR and the RSSR. The rest of the paper is structured as follows. The design principle of the proposed frequency 16 tupled structure is described in Section 2. The simulation results with the impact of non-ideal parameter variation on the SBSR are discussed in Section 3 and finally the conclusion is presented in Section 4 .

\section{DESIGN PRINCIPLE}

The MWPL is built using the OptiSystem version 17.0.0. Figure 1 shows a schematic diagram of the proposed filterless MM wave generation with frequency 16 tupling. For generating MM wave with FMF of 16, \pm eighth-order SBs must hit the photodetector (PD). In order to obtain this, MZM should be biased at MXTP which provides a carrier and only even order SBs after modulation. Furthermore, obtaining pure \pm eighth-order SBs is quite challenging because several other unwanted spurious SBs are also generated.

For suppressing all other spurious harmonics, subsequent measures are adopted in the proposed scheme as: 1) setting MZM at MXTP for suppressing all the odd order SBs and generating only even order SBs, 2) controlling the M.I. of the MZM to let the power of sidebands greater than \pm 10 th order sidebands return to zero or is negligible, and 3) adjusting the electrical phase shift of the RF driving signal between the MZM and insertion loss of modulator so as to suppress the carrier and eliminate all the other $(2 \mathrm{n}-2)^{\text {th }}$ order sidebands except \pm eighthorder SBs. The detailed principle of the proposed scheme is mentioned below.

It utilizes a laser diode (LD), an RF signal generator, electrical phase shifters, a photodiode, optical amplifiers, and a DDMZM. A continuous wave laser (CWL) with frequency $\omega_{c}$ emits the light signal which acts as a carrier signal defined as follows:

$$
V_{\text {in }}=V_{c}\left(\operatorname{Cos} \omega_{c} t\right) .
$$




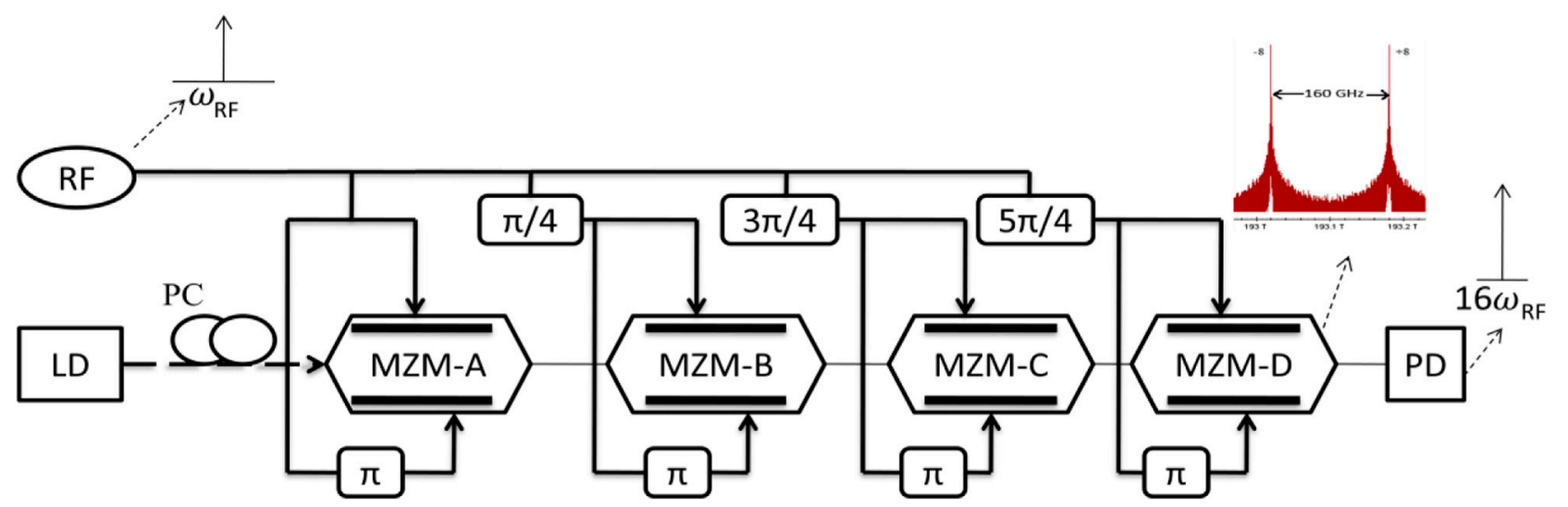

FIGURE 1 | Proposed structure for generating a 16-tupled MM wave signal.

Here, $V_{c}$ is the amplitude of the carrier signal.

This light carrier wave after being polarization controlled (PC) is transmitted to the first intensity modulator, that is, MZM-A triggered by RF LO which produces a single RF tone of frequency, $\omega_{r f}$, given by the following:

$$
V_{1}=V_{r f}\left(\operatorname{Cos} \omega_{r f} t\right) \text {, }
$$

where $V_{r f}$ is the amplitude of the input RF signal.

\section{Odd order Harmonics Elimination}

The transfer function of a DD-MZM is expressed as follows:

$$
E_{M Z M-A}(t)=\frac{\beta}{2} E_{c} e^{j \omega_{c} t}\left\{\chi e^{-j m_{a} \sin \omega_{R F} t}+(1-\chi) e^{j m_{a} \sin \omega_{R F} t}\right\},
$$

where $m_{a}=\frac{\pi V_{R F}}{V_{\pi}}$ is the M.I.,

$\mathrm{V}_{\mathrm{RF}}$ is the switching RF voltage, $\mathrm{V}_{\pi}$ is the half wave voltage of MZM, and $\beta$ is the insertion loss of MZM.

$\mathrm{x}=\frac{\left(1-\frac{1}{\sqrt{\mu_{r}}}\right.}{2}\left\{\mu_{r}=10^{(\text {MZM extinction ratio }) / 10}\right\}$ is the splitting ratio of upper and lower arms of MZM.

However, using the Jacobi-Anger expansion,

$$
\begin{aligned}
e^{j m s i n \Phi} & =\sum_{k=-\infty}^{k=\infty} J_{k}(m) e^{j k \Phi} \text { and } e^{j m \cos \Phi} \\
& =\sum_{k=-\infty}^{k=\infty}(-1)^{k} J_{k}(m) e^{j k \Phi} .
\end{aligned}
$$

The transfer function can be rewritten as follows:

$$
\begin{aligned}
E_{M Z M}(t) & =\beta E_{c}\left[\sum_{k=-\infty}^{k=\infty}(-1)^{k} J_{k}(m) e^{j\left\{\left(\omega_{c}+k \omega_{R F}\right) t\right\}}\right], \\
E_{M Z M}(t)= & \beta E_{c}\left[J_{0}\left(m_{a}\right) e^{j \omega_{c} t}-J_{1}\left(m_{a}\right)\left\{e^{j\left(\omega_{c}-\omega_{R F}\right) t}+e^{j\left(\omega_{c}+\omega_{R F}\right) t}\right\}\right. \\
& +J_{2}\left(m_{a}\right)\left\{e^{j\left(\omega_{c}-2 \omega_{R F}\right) t}+e^{j\left(\omega_{c}+2 \omega_{R F}\right) t}\right\} \\
& \left.-J_{3}\left(m_{a}\right)\left\{e^{j\left(\omega_{c}-3 \omega_{R F}\right) t}+e^{j\left(\omega_{c}+3 \omega_{R F}\right) t}\right\}+\ldots \ldots \ldots\right] .
\end{aligned}
$$

As the $\mathrm{Li}-\mathrm{NbO}_{3}$ DD-MZM has a nonlinear characteristic response, the modulated output optical signal consists of a carrier along with multiple sidebands symmetrically located around it as depicted in Eq. 6. The amplitude and number of sidebands generated around the carrier can be controlled by varying $m_{a}$ which in turn depends on $\mathrm{V}_{\mathrm{RF}}$ and $\mathrm{V}_{\pi}$. In order to suppress the odd order SBs, the first modulator is biased at MXTP by applying the bias voltages to the upper and lower arms of the modulator such that $V_{\text {bias }}=V_{\text {bias } 1}-V_{\text {bias } 2}=0$ with a $\pi$ phase difference between the RF signals fed to the arms of the modulator.

$$
\begin{aligned}
E_{M Z M-A}(t)= & \beta E_{c} e^{j \omega_{c} t}\left[J_{0}\left(m_{a}\right)+J_{2}\left(m_{a}\right)\left\{e^{j 2 \omega_{R F} t}+e^{-j 2 \omega_{R F} t}\right\}\right. \\
& +J_{4}\left(m_{a}\right)\left\{e^{j 4 \omega_{R F} t}+e^{-j 4 \omega_{R F} t}\right\}+J_{6}\left(m_{a}\right)\left\{e^{j 6 \omega_{R F} t}\right. \\
& \left.+e^{-j 6 \omega_{R F} t}\right\}+J_{8}\left(m_{a}\right)\left\{e^{j 8 \omega_{R F} t}+e^{-j 8 \omega_{R F} t}\right\} .
\end{aligned}
$$

The above equation clearly depicts that there are $2 \mathrm{n}^{\text {th }}$ order, that is, even SBs generated from the first modulator, and the corresponding optical spectrum is shown in Figure 2.

\section{Suppressing $(2 n-2)^{\text {th }}$ Order Sidebands}

For suppressing $(2 \mathrm{n}-2)^{\text {th }}$ order sidebands, except the desired \pm eighth sidebands, an MZM-A output is fed to the second one, which in turn goes to the third and then to the fourth in such a manner that all the modulators are driven with the RF signal with a suitable phase shift between the two consecutive modulators such that the output from the last modulator only contains the desired \pm eighth-order SB and the separation between the desired sidebands is the 16th multiple of input RF.

The modulated output from MZM-A is sent to MZM-B which is triggered by an RF signal with a $45^{\circ}$ phase difference such that $V_{2}=V_{r f}\left(\operatorname{Cos} \omega_{r f} t+\pi / 4\right)$ is mathematically expressed in Eq. 6 as follows: 


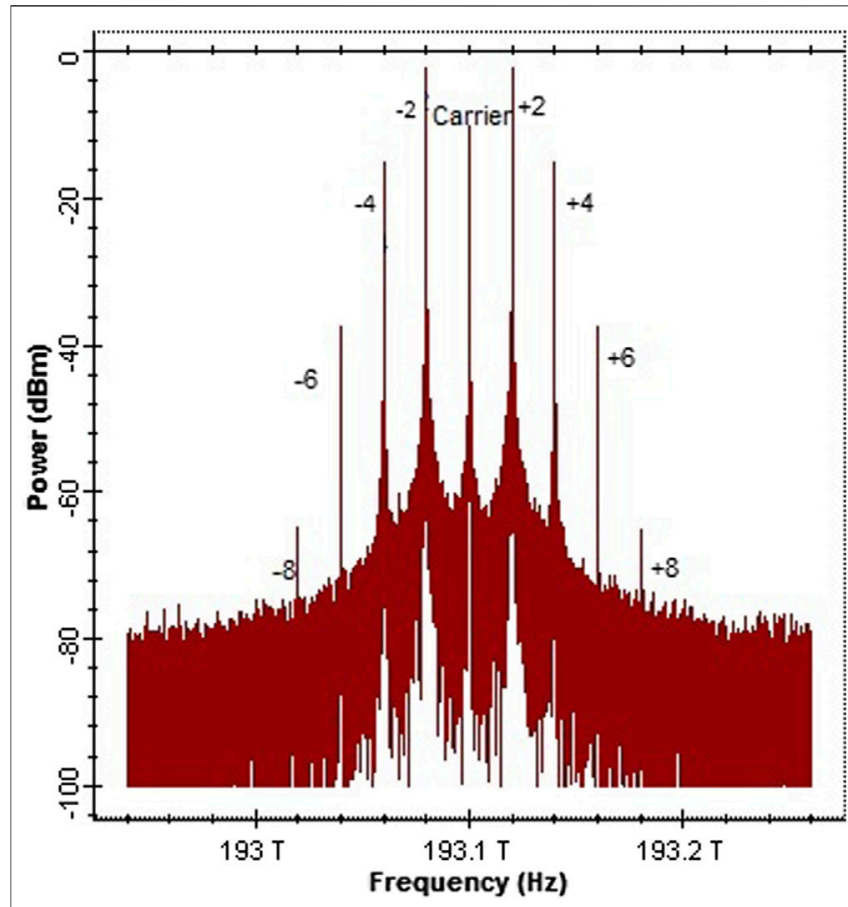

FIGURE 2 | Optical spectrum output from MZM-A containing only even order sidebands.

$$
E_{M Z M-B}(t)=\frac{\beta^{2}}{2} E_{c} e^{j \pi / 4}\left[\sum_{k=-\infty}^{k=\infty} J_{k}^{2}\left(m_{a}\right) e^{j\left\{\left(\omega_{c}+2 k \omega_{R F}\right) t+k \pi\right\}}\right] .
$$

This output from MZM-B is an input to the third MZM triggered by the RF signal with an electrical phase difference of $135^{\circ}$

$$
\begin{aligned}
E_{M Z M-c}(t)= & \beta E_{M Z M-B}(t)\left\{J_{0}\left(m_{a}\right)-J_{2}\left(m_{a}\right)\right\}\left\{e^{j 2 \omega_{R F} t}+e^{-j 2 \omega_{R F} t}\right\} \\
& +J_{4}\left(m_{a}\right)\left\{e^{j 4 \omega_{R F} t}+e^{-j 4 \omega_{R F} t}\right\}-J_{6}\left(m_{a}\right)\left\{e^{j 6 \omega_{R F} t}\right. \\
& \left.+e^{-j 6 \omega_{R F} t}\right\}+J_{8}\left(m_{a}\right)\left\{e^{j 8 \omega_{R F} t}+e^{-j 8 \omega_{R F} t}\right\} \\
& -J_{10}\left(m_{a}\right)\left\{e^{j 10 \omega_{R F} t}+e^{-j 10 \omega_{R F} t}\right\}+J_{12}\left(m_{a}\right)\left\{e^{j 12 \omega_{R F} t}\right. \\
& \left.+e^{-j 12 \omega_{R F} t}\right\} .
\end{aligned}
$$

This output is fed to the last modulator, that is, MZM-D which is driven by the RF signal phase shifted by $225^{\circ}$. By providing this phase shift, $J_{2}\left(m_{a}\right), J_{4}\left(m_{a}\right)$, and $J_{6}\left(m_{a}\right)$ terms turn into zero and other higher order harmonics, such as $J_{10}\left(m_{a}\right)$ and $J_{12}\left(m_{a}\right)$, have extremely low power which can be neglected.

\section{Tunable Optical Carrier Suppression}

By properly adjusting the modulation index, the carrier could be suppressed completely which results into power saving and efficient transmission leaving behind the optical spectrum with only the eighth-order $\mathrm{SB}$ as depicted in Figure 3. The MZM-D output can be stated mathematically as follows:

$$
E_{M Z M-D}(t)=\frac{\beta^{4}}{2} J_{8}^{2}\left(m_{a}\right) E_{c} e^{j \omega_{c} t}\left\{e^{j 8 \omega_{R F} t}+e^{-j 8 \omega_{R F} t}\right\} .
$$

This output is then detected at the receiver side through the PIN photodiode whose photocurrent is given by the following equation:

$$
\begin{gathered}
I_{p h}(t)=\mu_{p h}\left|E_{M Z M-D}(t)\right| *\left|E_{M Z M-D}(t)\right|^{*}, \\
I_{p h}(t)=\mu_{p h}\left\{\frac{\beta}{2} E_{c}\left[J_{-8}\left(m_{a}\right) e^{j\left(\omega_{c}-8 \omega_{R F}\right) t}+J_{8}\left(m_{a}\right) e^{j\left(\omega_{c}+8 \omega_{R F}\right) t}\right]\right\} \\
*\left\{\frac{\beta}{2} E_{c}\left[J_{-8}\left(m_{a}\right) e^{j\left(\omega_{c}-8 \omega_{R F}\right) t}+J_{8}\left(m_{a}\right) e^{j\left(\omega_{c}+8 \omega_{R F}\right) t}\right]\right\},
\end{gathered}
$$

where $\mu_{p h}$ is the responsivity of the photodiode. The above equation can be further simplified as follows:

$$
I_{p h}(t)=\frac{\mu_{p h} \beta^{2}\left|E_{M Z M-D}(t)\right|^{2}}{4} \operatorname{Cos}\left(16 \omega_{R F} t\right) .
$$

Thus, it can be seen mathematically through Eq. 13 that the photo-detected output consists of an electrical signal of frequency $160 \mathrm{GHz}$ as shown in the Figure 4 which is 16 times the RF signal frequency, that is, $10 \mathrm{GHz}$ being utilized at the CS.

\section{RESULTS AND DISCUSSION}

A polarization-controlled CWL emitting a light signal of frequency $193.1 \mathrm{THz}$ is sent to MZM-A biased at MXTP and triggered through a $10 \mathrm{GHz}$ RF signal. As MZM-A is biased at MXTP, its output contains only even order sidebands. The output from the first modulator is sent to the second, then to the third,

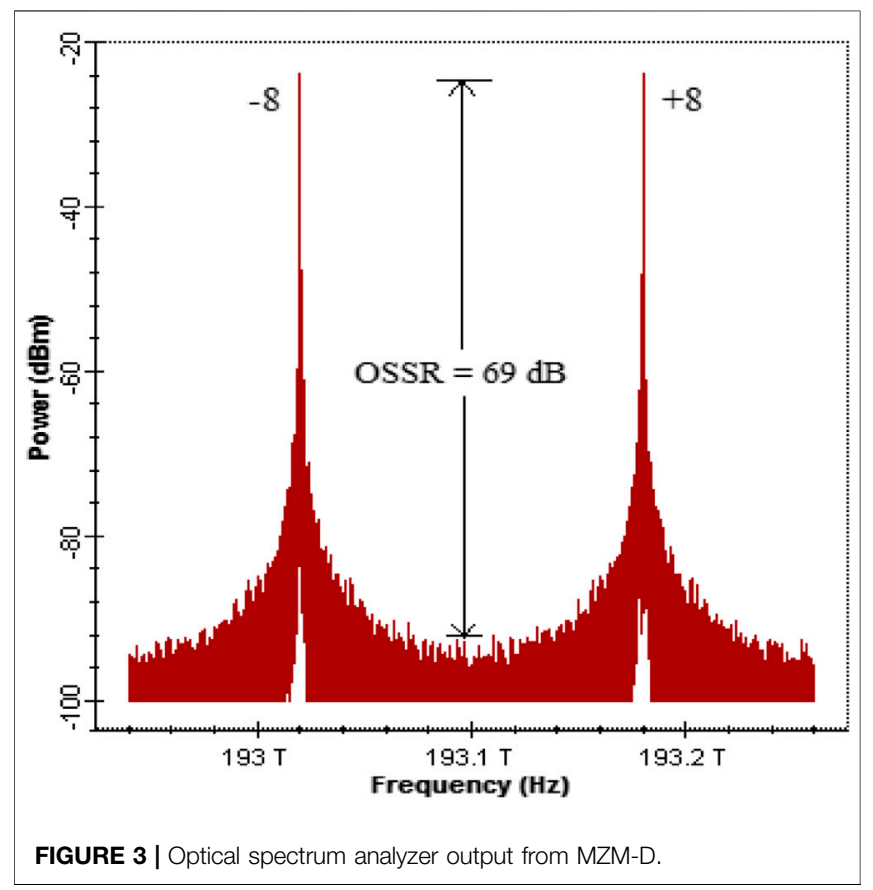


TABLE 2 | Simulation parameters employed for the present scheme of frequency $16 \mathrm{MM}$ wave signal generation.

\begin{tabular}{lll} 
System block & \multicolumn{1}{c}{ Simulation parameters } & Parameters value \\
\hline Fiber link & Length & $60 \mathrm{~km}$ \\
& Attenuation & $0.22 \mathrm{~dB} / \mathrm{km}$ \\
& Dispersion & $16.75 \mathrm{ps} / \mathrm{nm}-\mathrm{km}$ \\
CW laser Diode & Dispersion slope & $0.075 \mathrm{ps} / \mathrm{nm}^{2} / \mathrm{k}$ \\
& Frequency & $193.1 \mathrm{THz}$ \\
Local oscillator & Power & $8 \mathrm{dBm}$ \\
MZM modulator & RF signal & $10 \mathrm{GHz}$ \\
& MZM-A, B, and C extinction ratio & $60 \mathrm{~dB}$ \\
& MZM-D extinction ratio & $55 \mathrm{~dB}$ \\
& Insertion loss & $3 \mathrm{~dB}$ \\
EDFA 1 & Switching bias voltage & 3.2 \\
& Switching RF voltage & 3.2 \\
Amplifier & Gain & $10 \mathrm{~dB}$ \\
& Noise figure (NF) & $3 \mathrm{~dB}$ \\
PIN photodiode & Gain & $12 \mathrm{~dB}$ \\
& Noise figure & $2 \mathrm{~dB}$ \\
& PIN photodiode responsivity & 0.9 \\
& PIN dark current & $9 \mathrm{nA}$ \\
& &
\end{tabular}

and at last to the fourth modulator in such a manner that the electrical phase shift of the RF local oscillator (LO) signal between first two consecutive MZMs is $45^{\circ}$ and next two MZMs is $90^{\circ}$, and all the four modulators are biased at MXTP. By arranging the electrical phase shift, the modulation index, and the MZM in this manner, the output from the last modulator suppresses the carrier with all other spurious harmonics and contains only eighth-order SBs at $\omega_{\mathrm{c}}+8 \omega_{\mathrm{RF}}$, that is, $193.18 \mathrm{THz}$, and $\omega_{\mathrm{c}}$ $-8 \omega_{\mathrm{RF}}$, that is, $193.02 \mathrm{THz}$ with $-22.4 \mathrm{dBm}$ power. It can be

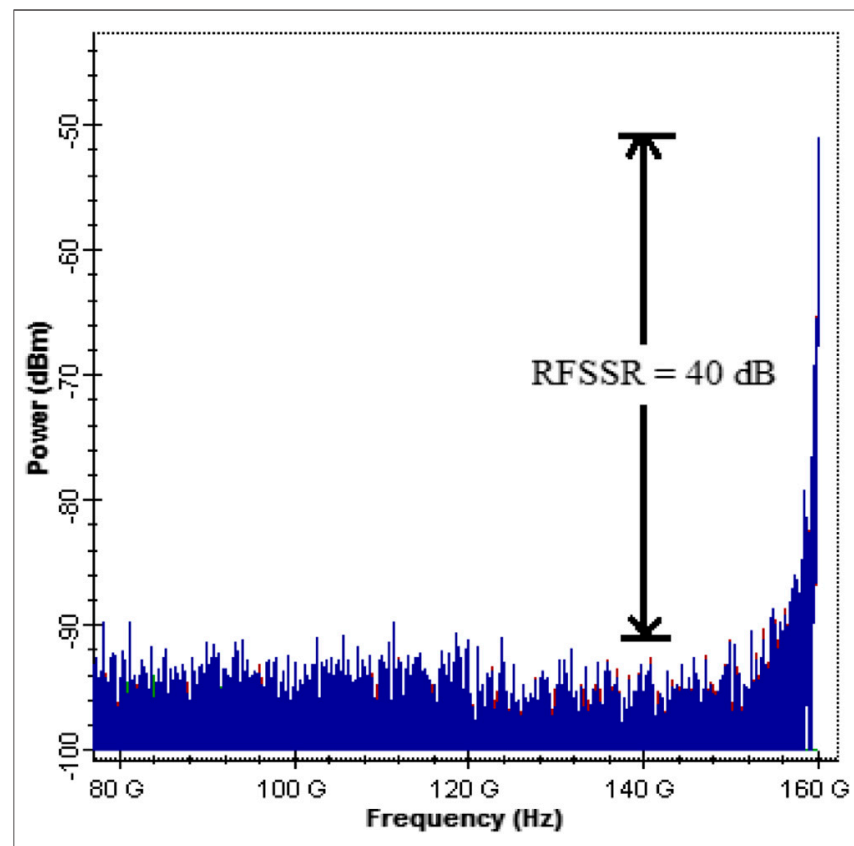

FIGURE 4 | RF spectrum of the photo-detected signal from the PIN photodiode. seen that the difference between +eighth $(193.18 \mathrm{THz})$ - and -eighth (193.02 THz)-order SBs is the 16th multiple of the input electrical signal. These sidebands are then sent to the BS through a $60-\mathrm{km}$ standard single-mode fiber (SSMF) with parameters mentioned in Table 2. At the BS, a hybrid configuration of cascaded amplifiers consisting of EDFA with gain of $10 \mathrm{~dB}$ and optical amplifier having $15 \mathrm{~dB}$ gain is utilized to mitigate the losses incurred during transmission over the fiber, and then a PIN photodiode with a responsivity of $0.9 \mathrm{~A} / \mathrm{W}$ is utilized to detect the modulated signal by converting it back to electrical form and hence a $160-\mathrm{GHz}$ signal is detected using the RF spectrum analyzer with tan output power of $-50 \mathrm{dBm}$.

\section{Impact of Nonideal Parameters on the OSSR and the RSSR}

The above-mentioned results are based on the simulation carried over the OptiSystem software by assuming ideal parameters as mentioned in Table 2. However, these parameters might deviate from their desired values and thus it is crucial to analyze the impact of several nonideal parameters, such as RF voltage variation, the MZM extinction ratio, a cascaded amplifier configuration, and phase drift is analyzed over the OSSR and the RSSR.

\section{Extinction Ratio and Splitting Ratio}

For evaluating the proposed system performance, the impact of the extinction ratio (E. R.) or the splitting ratio of each MZM is studied over the OSSR and the RSSR, as shown in Figure 5. It is observed that as the extinction ratio varies from its ideal value, the system is not able to suppress the odd order SBs as $\mathrm{P} 8 / \mathrm{P} 1$ is $57 \mathrm{~dB}$ when it deviates by 0.010 from the ideal value with $30 \mathrm{~dB}$ RSSR, and the eighth-order SBs are $26 \mathrm{~dB}$ greater than seventh order SBs when it deviates by a value of 0.045 with the 16 -tupled MM wave being only $13 \mathrm{~dB}$ higher than the 15-tupled signal deteriorating the system performance, as shown in Figure 5. This states that the OSSR and the RSSR rises with an increasing splitting ratio/ extinction ratio, as the splitting ratio is dependent on the extinction ratio of the MZM. Both the sideband suppression ratio (SSR) are maximum at 0.4995 splitting ratio, as calculated from Eq. 3 under Odd Order Harmonics Elimination, and the unwanted sidebands are completely eliminated when the MZM extinction ratio is set to $60 \mathrm{~dB}$ being independent of the extinction ratio exceeding $60 \mathrm{~dB}$, as clearly depicted in Figures 6, 7 .

\section{Modulation Index}

The optical and RF spectrum when M.I. deviates 0.0588 from the ideal value is depicted in Figure 8 where the $\mathrm{P}_{8}$ is $14 \mathrm{~dB}$ higher than $\mathrm{P}_{0}$ and $62 \mathrm{~dB}$ from $\mathrm{P}_{3}$, respectively. However, the 16-tupled $\mathrm{MM}$ wave is $11 \mathrm{~dB}$ greater than the octupling wave (eighth multiple of RF), which is quite less for the applications requiring $\mathrm{MM}$ wave for their operation. Besides this, the impact of the modulation index on the sideband power ratio $(\mathrm{SBPR})\left(\mathrm{P}_{8} / \mathrm{P}_{0}\right)$ is depicted in Figure 9 , which shows that the system provides $\left(\mathrm{P}_{8} / \mathrm{P}_{0}\right)$ greater than $5 \mathrm{~dB}$ 

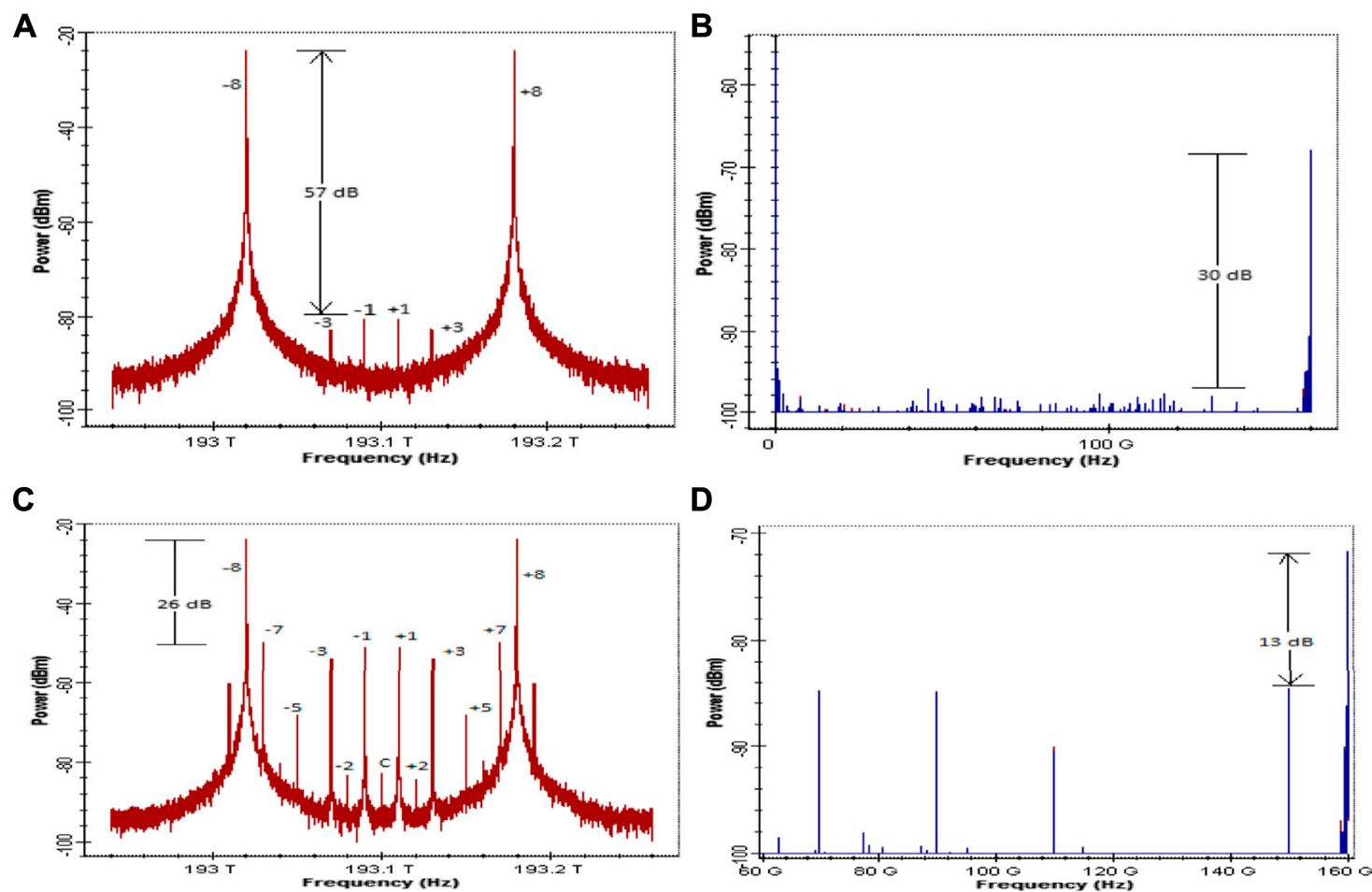

FIGURE 5 | (A) Optical spectrum when E.R. deviates by 0.010, (B) RF spectrum when E.R. deviates by 0.010, (C) optical spectrum when E.R. deviates by 0.045, and (D) RF spectrum when E.R. deviates by 0.045 value.

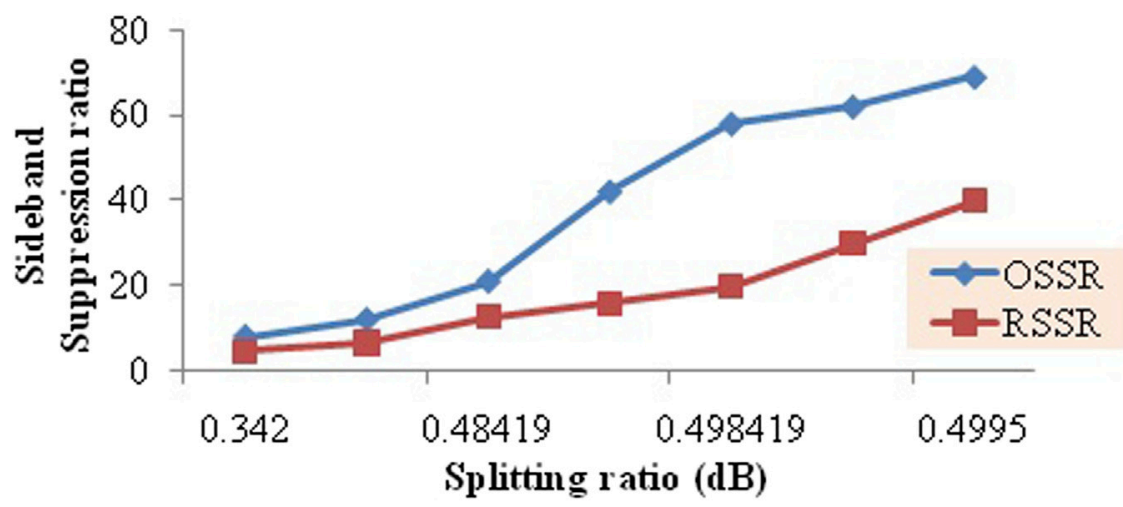

FIGURE 6 | Impact of the splitting ratio on the sideband suppression ratio.

and the RSSR greater than $2 \mathrm{~dB}$ (Figure 10) in the M.I. range of $2.678-2.945$ by providing the peak of the OSSR $\left\{\right.$ i.e., $\left.\left(\mathrm{P}_{8} / \mathrm{P}_{0}\right)\right\}$ and the RSSR at an M.I. of 2.8258 with an RF voltage of 2.87989 . The system provides an acceptable and tunable SSR which is greater than $15 \mathrm{~dB}$ in the M.I. ranging from 2.778 to 2.873 .

\section{Phase Drift Analysis of the OSSR}

The effect of phase drift can be analyzed over the system by studying the OSSR deviation over the phase drift in PS. It can be noticed that for $\pm 10^{\circ}$ phase drift between MZM upper arm and lower arms OSSR is above $25 \mathrm{~dB}$ which means that the system is less sensitive as far as MZM arms' phase drift is considered, while it is sensitive toward the phase drift in the phase shifters of the RF signal which drives the DD-MZM modulator, as depicted in Figure 11. However, the system provides acceptable performance for $\pm 8^{\circ}$ phase drift.

\section{Cascaded Hybrid Amplifier Configuration}

Amplifiers are used in the system so as to compensate the losses which are inserted while the signal travels through the optical 


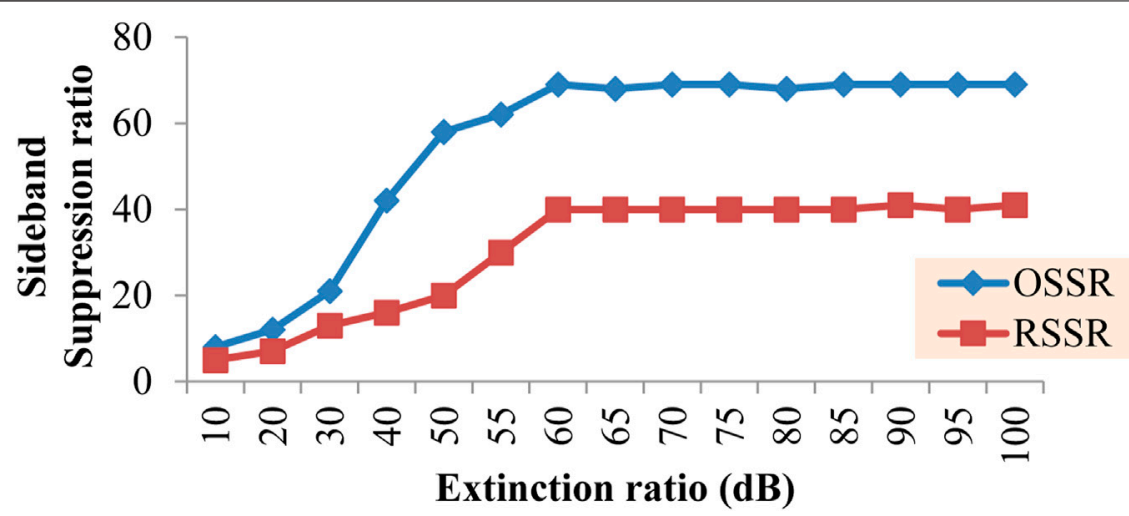

FIGURE 7 | Impact of the MZM extinction ratio on the sideband suppression ratio.
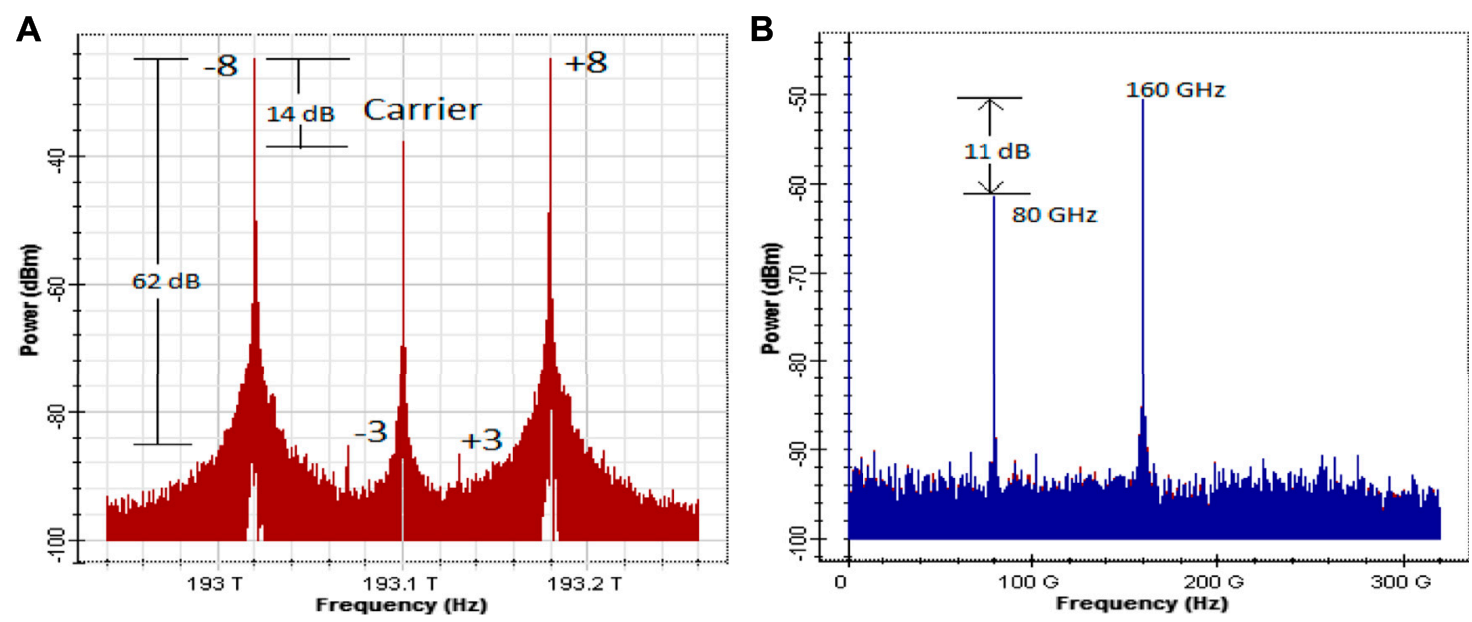

FIGURE 8 | (A) Optical spectrum and (B) RF spectrum output at M. I of 2.776.

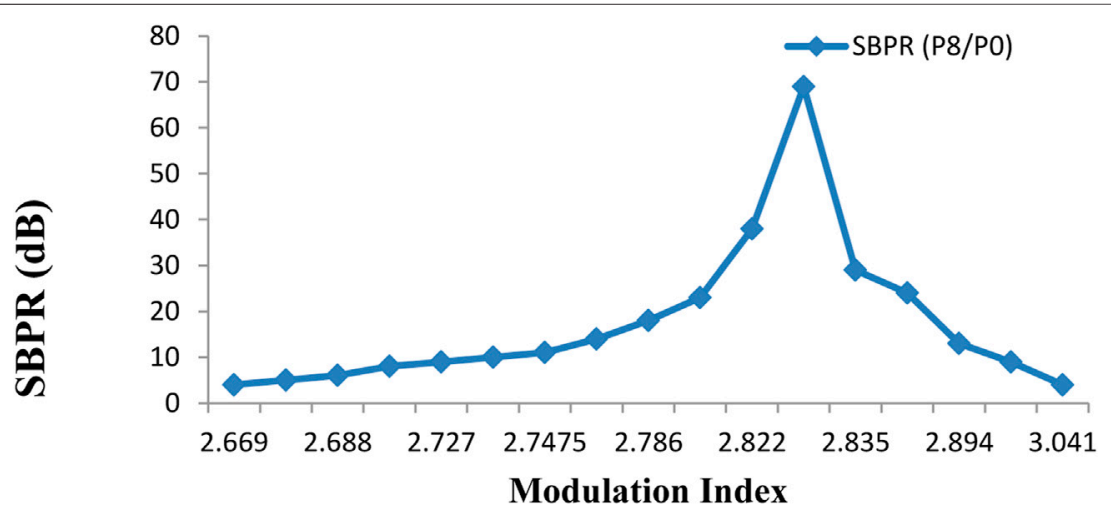

FIGURE 9 | SBPR variation with the modulation index. 


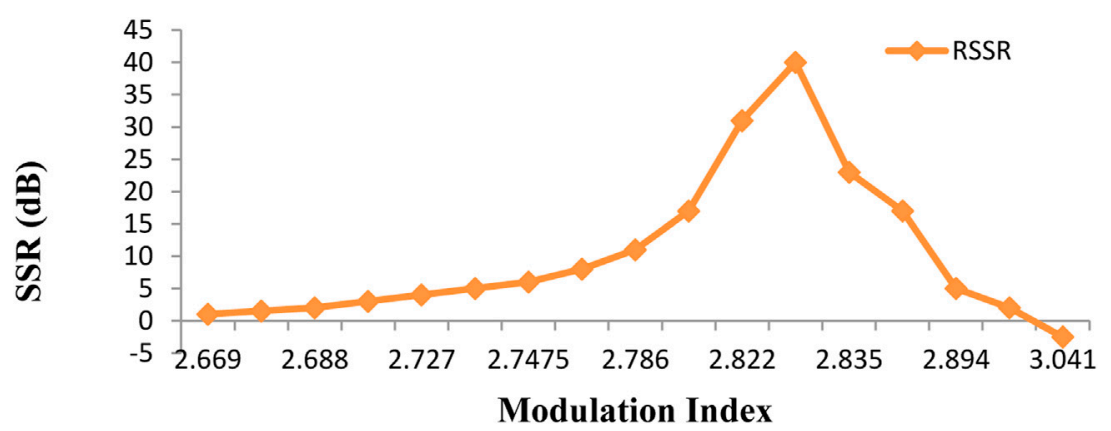

FIGURE 10 | RSSR variation with the modulation index.

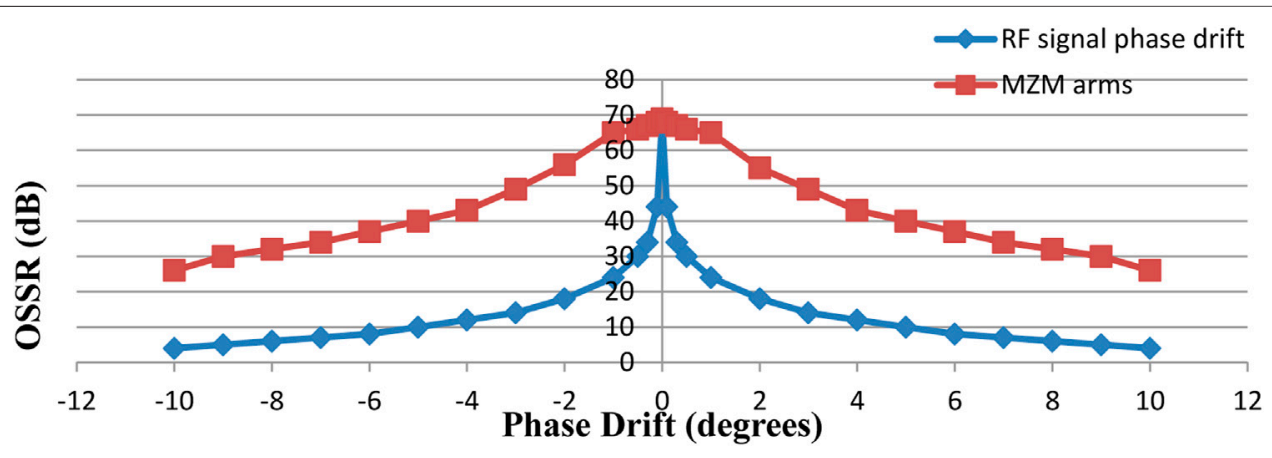

FIGURE 11 | OSSR variation with phase drift in the RF signal driving MZM and MZM arms.
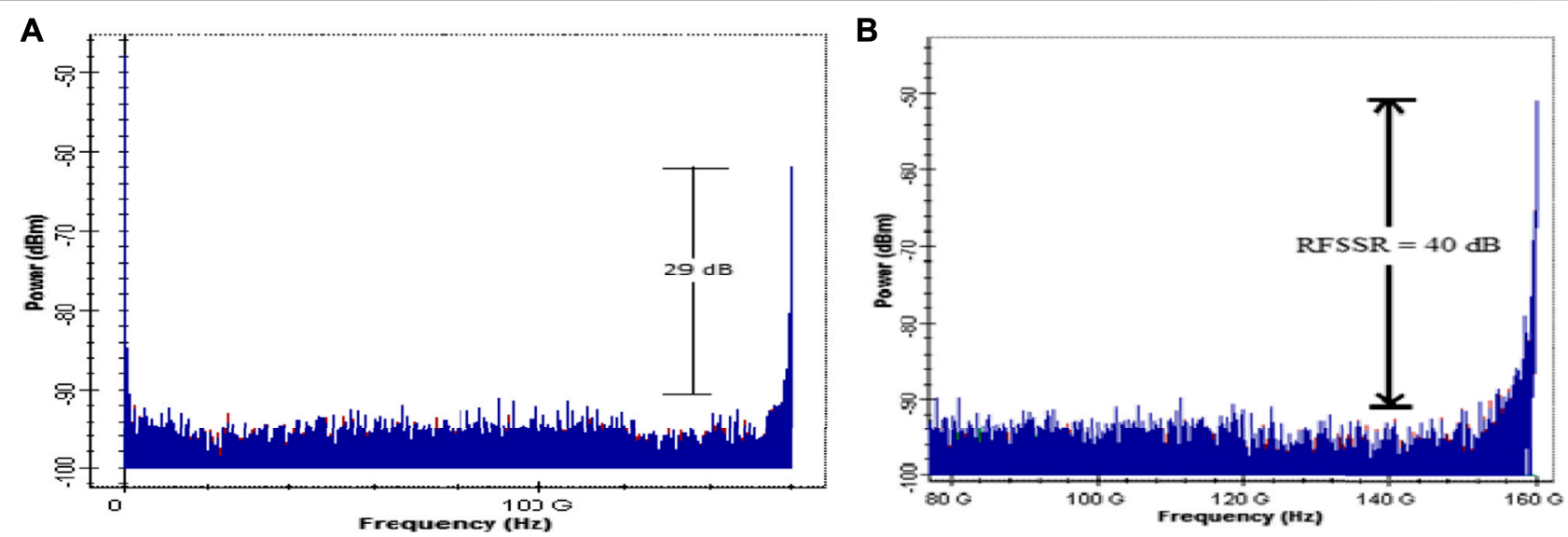

FIGURE 12 | RSSR for the system with (A) a single amplifier and (B) a cascaded amplifier.

fiber. The impact of using a cascaded amplifier configuration having EDFA 1 connected with optical amplifier with their parameters mentioned in Table 2 enhances the RFSSR by $11 \mathrm{~dB}$ in comparison to a single amplifier configuration with a
20- $\mathrm{dB}$ gain and NF of $4 \mathrm{~dB}$ when detected by the PIN photodiode generates a $160-\mathrm{GHz}$ with a 29-dB RFSSR, as shown in Figure 12. It can be noted that the cascaded amplifier configuration results in the improved RFSSR. 


\section{CONCLUSION}

A photonic generation scheme of a frequency 16-tupled MM wave signal is presented and demonstrated using a seriesconnected DD-MZM with a double sideband optical carrier suppression modulation offering efficient power transmission. With appropriate adjustment of internal parameters, such as the modulation index, DC biasing, the splitting ratio, and electrical phase difference, and utilizing a hybrid amplifier configuration, a $160-\mathrm{GHz}$ MM wave signal is generated from a $10-\mathrm{GHz}$ RF signal with an OSSR of $69 \mathrm{~dB}$ and an RSSR of $40 \mathrm{~dB}$. This system does not require any additional circuitry such as a mixer, a filter, and a digital signal processor. In comparison to the previous approaches, the proposed structure optically generates a highly conversant, spectrally pure 16-tupled MM wave signal with a better tunable OSSR and an RSSR and with a wider tunable range from $1-15 \mathrm{GHz}$, as there is no filter required in this scheme. Further, the system provides

\section{REFERENCES}

1. Zeb K. Photonic generation of spectrally pure Millimeter wave signals for $5 G$ applications. Ottawa, Canada: IEEE International Topical Meeting on Microwave Photonics (2020). p. 1-4.

2. Perez Galacho D, Sartiano D, and Sales $S$. Analog radio over fiber links for future $5 G$ radio access networks. IEEE ICTON (2019) 1-4. doi:10.1109/icton.2019.8840516

3. He X. Linear analog photonic link enabled RoF-5G front haul transmission system. Optik (2019) 183(3):99-104. doi:10.1016/j.ijleo.2019.02.056

4. Kamissoko D, Jing $H$, Ganame $H$, and Tall $M$. Performance investigation of W-band Millimeter wave radio over fiber system employing optical heterodyne generation and self homodyne detection. Opt Commun (2020) 474(1):1-8. doi:10.1016/j.optcom.2020.126174

5. Asha SD. A Comprehensive Review of Millimeter wave based radio over fiber for $5 \mathrm{G}$ front haul transmissions. Ijst (2021) 14(1):86-100. doi:10.17485/ijst/v14i1.2177

6. Zhu Z, Zhao S, Chu X, and Dong Y. Optical generation of millimeter-wave signals via frequency 16-tupling without an optical filter. Opt Commun (2015) 354(1):40-7. doi:10.1016/j.optcom.2015.05.035

7. Li X, Zhao S, Zhu Z, Gong B, Chu X, Li Y, et al. An optical millimeter-wave generation scheme based on two parallel dual-parallel Mach-Zehnder modulators and polarization multiplexing. J Mod Opt (2015) 62(18): 1502-9. doi:10.1080/09500340.2015.1045948

8. Ying X, Xu T, Li W, Weng H, and Zhang X. Photonic generation of millimeterwavesignal via frequency 16-tupling based on cascaded dual - parallel MZM. J Optoelectronics - Laser (2017) 28(11):1212-7.

9. Dar AB, Ahmad F, and Jha RK. Filterless 16-Tupled Optical Millimeter-wave Generation Using Cascaded Parallel Mach-zehnder Modulators with Extinction Ratio Tolerance. PIER Lett (2020) 91(1):129-35. doi:10.2528/pierl20031009

10. Muthu K, Raja A, and Shanmugapriya G. Frequency16-tupled optical millimeter wavegeneration using dual cascaded MZMs and 2.5 Gbps RoF tr ansmission. Optik (2017) 14(1):338-46. acceptable performance with a wider range of M.I. variation $2.778-2.873, \pm 8^{\circ}$ phase drift between MZM arms, and electrical phase shifters and is independent of the extinction ratio beyond $60 \mathrm{~dB}$.

\section{DATA AVAILABILITY STATEMENT}

The original contributions presented in the study are included in the article/Supplementary Material; further inquiries can be directed to the corresponding author.

\section{AUTHOR CONTRIBUTIONS}

SD proposed the idea and supervised the whole work. Asha carried out the modeling and simulation. Both authors contributed to the writing of the manuscript.

11. Esakki Muthu K, and Sivanantha Raja A. Bidirectional MM-Wave Radio over Fiber transmission through frequency dual 16-tupling of RF local oscillator. J Eur Opt Soc - Rapid Publications. (2016) 12(24):1-9. doi:10.1186/s41476016-0028-2

12. Chen H, Ning T, Jian W, Pei L, and Li J. D-band millimeter-wave generator based ona frequency 16-tupling feed-forward modulation technique. Opt Eng (2013) 52(1):0761041-4. doi:10.1117/1.oe.52.7.076104

13. Wang D, Tang X, Xi L, Zhang X, and Fan Y. A filterless scheme of generating frequency 16-tupling millimeter-wave based on only two MZMs. Opt Laser Tech (2019) 116(1):7-12. doi:10.1016/ j.optlastec.2019.03.009

14. Baskaran M, and Prabakaran R. Optical Millimeter wave signal generation with frequency 16 tupling using cascaded MZMs and no optical filtering for radio over fiber system. J Eur Opt Society-Rapid Publications (2018) 14(13): 1-8. doi:10.1186/s41476-018-0080-1

Conflict of Interest: The authors declare that the research was conducted in the absence of any commercial or financial relationships that could be construed as a potential conflict of interest.

Publisher's Note: All claims expressed in this article are solely those of the authors and do not necessarily represent those of their affiliated organizations, or those of the publisher, the editors and the reviewers. Any product that may be evaluated in this article, or claim that may be made by its manufacturer, is not guaranteed or endorsed by the publisher.

Copyright (C) 2021 Asha and Dahiya. This is an open-access article distributed under the terms of the Creative Commons Attribution License (CC BY). The use, distribution or reproduction in other forums is permitted, provided the original author(s) and the copyright owner(s) are credited and that the original publication in this journal is cited, in accordance with accepted academic practice. No use, distribution or reproduction is permitted which does not comply with these terms. 